\title{
Efecto diurético de la especie Salvia scutellarioides en ratas
}

\author{
Jorge Hernán Ramírez, Mauricio Palacios, Oscar Gutiérrez
}

Laboratorio de Farmacología, Departamento de Ciencias Fisiológicas, Facultad de Salud, Universidad del Valle, Cali, Colombia.

Introducción. En la medicina tradicional colombiana se usa la infusión de toda la planta llamada "mastranto" u "oreja de perro" (Salvia scutellarioides) por sus efectos antihipertensivos y diuréticos. Hasta ahora no se han hecho estudios experimentales para validar los efectos reportados.

Objetivos. Determinar el efecto de S. scutellarioides en la diuresis y concentración de electrolitos urinarios utilizando un modelo en ratas.

Materiales y métodos. Veinticuatro ratas Sprague-Dawley machos fueron repartidas al azar en cuatro grupos homogéneos: el grupo 1 recibió solución salina normal; el grupo 2, furosemida (10 mg/kg), y los grupos 3 y 4 , S. scutellarioides (1 g/kg y $2 \mathrm{~g} / \mathrm{kg}$, respectivamente). Todos los tratamientos se administraron en un volumen de $25 \mathrm{ml} / \mathrm{kg}$ de peso del animal. Las ratas se colocaron en una jaula metabólica durante seis horas, y se cuantificó la excreción urinaria y los electrolitos en orina.

Resultados. La administración de $S$. scutellarioides en dosis de 1 y $2 \mathrm{~g} / \mathrm{kg}$ produjo un aumento significativo de la diuresis comparado con la del grupo control $(p<0,01)$. El efecto diurético se manifestó principalmente a partir de la cuarta hora de administración de S. scutellarioides. La administración de $S$. scutellarioides en ambas dosis produjo un incremento en la excreción urinaria de potasio y cloro.

Conclusiones. El estudio corrobora la aparente actividad diurética de $S$. scutellarioides reportada por médicos tradicionales, lo que podría explicar su posible efecto antihipertensivo. Se requieren más estudios para determinar el perfil farmacológico y la toxicidad de la planta.

Palabras clave: diuréticos, Lamiaceae, Salvia, etnofarmacología, fitoterapia, medicina herbaria.

Diuretic effect of an infusion of the herbal plant, Salvia scutellarioides, in rats.

Introduction. In the Colombian traditional medicine, an infusion made of the entire plant Salvia scutellarioides (known locally as mastranto or oreja de perro (dog's ear)) is used for its antihypertensive and diuretic effects. However, experimental studies have never been done to validate the reported effects.

Objective. In the current study, a rat model was used to determine the effect of $S$. scutellarioides on rat diuresis and urinary electrolytes concentration.

Materials and methods. Twenty-four male Sprague-Dawley rats were distributed in four groups: Group 1-normal saline solution, Group 2-furosemide $(10 \mathrm{mg} / \mathrm{kg})$, Groups 3 and 4 with $S$. scutellarioides infusion, $1 \mathrm{~g} / \mathrm{kg}$ and $2 \mathrm{~g} / \mathrm{kg}$ respectively. All treatments were administered in a volume of $25 \mathrm{ml} / \mathrm{kg}$ of rat weight. After treatment, the rats were placed in a metabolic cage for 6 hours. During this period, urinary excretion was monitored and quantified. At the end of 6 hours, the urinary electrolyte concentrations were measured.

Results. The administration of $S$. scutellarioides at doses of 1 and $2 \mathrm{~g} / \mathrm{kg}$ produced a significant increase in diuresis when compared to the control group $(p<0.01)$. The administration of $S$. scutellarioides at these doses also increased the urinary excretion of potassium and chloride. Conclusions. These results corroborate the apparent diuretic activity of $S$. scutellarioides described by traditional herbalists and possibly explains its reported antihypertensive effect. Further studies are required to determine the pharmacological and toxicological profile of the plant.

Keywords: Diuretics, herbal medicine, Lamiaceae, Salvia, ethnopharmacology, phytotherapy. 
La especie Salvia scutellarioides (Lamiaceae sinonimia: S. paleafolia) es una planta autóctona de Colombia, de crecimiento endémico en las regiones pacífico y andina, donde es comúnmente conocida por la población como "mastranto". La infusión de los tallos y hojas de $S$. scutellarioides se emplea ampliamente en la medicina tradicional colombiana por sus efectos diuréticos y antihipertensivos (1-4).

Los análisis fitoquímicos realizados en $S$. scutellarioides han reportado que la planta presenta diferentes tipos de alcaloides, triterpenos, lignanos y flavonoides, pero hasta el momento no se han caracterizado sus propiedades farmacológicas in vitro ni in vivo (5). Pese al amplio uso de $S$. scutellarioides en la medicina tradicional colombiana, no se han realizado estudios de esta planta de diuresis o hipertensión en modelos animales. Se han descrito moléculas con efecto en músculo liso vascular en plantas de la misma familia y especie, algunas de las cuales se están evaluando en fases de experimentación preclínica y clínica (6-8).

El presente estudio evalúa el efecto de la administración de $S$. scutellarioides en la diuresis y excreción de electrolitos urinarios utilizando un modelo de ratas.

\section{Materiales y métodos}

\section{Obtención de la planta y almacenamiento}

Las muestras de $S$. scutellarioides se recolectaron en el kilómetro 18 de la vía a Buenaventura en un mercado local de plantas medicinales. La identificación de la planta la realizó Phillipe Silverstone (biólogo botánico, profesor titular de Biología, Facultad de Ciencias, Universidad del Valle); las muestras de hojas, tallos y raíces de $S$. scutellarioides se almacenaron en el herbario del Departamento de Biología de la Universidad del Valle.

Correspondencia:

Jorge Hernán Ramírez, Universidad del Valle, sede San Fernando, Calle 4 B \# 36-00, Edificio 116, Farmacología. Cali, Valle del Cauca.

Telefax: 092: 5185620, y 5542481

jorgehramirez31@yahoo.com

Recibido: 09/06/05; aceptado: 23/12/05
El material recolectado se lavó y, posteriormente, se desecó en un horno de flujo laminar a $35^{\circ} \mathrm{C}$ durante 48 horas. El almacenamiento se realizó en envases cerrados a una temperatura de $0^{\circ} \mathrm{C}$. Los procesos empleados para la recolección y procesamiento de la planta se realizaron de acuerdo a las recomendaciones del CYTED para la recolección y procesamiento de plantas medicinales (9).

\section{Preparación del extracto}

Las hojas y tallos secos se fraccionaron mecánicamente; para cada preparación del extracto se tomaron $10 \mathrm{~g}$ del material y se les practicó un proceso de extracción con arrastre de vapor en $500 \mathrm{ml}$ de agua destilada.

El extracto acuoso obtenido se colocó en un rotoevaporador hasta obtener un volumen de 25 $\mathrm{ml}$, equivalente a un extracto acuoso al $40 \%$ de concentración.

\section{Animales de experimentación}

El modelo experimental se llevó a cabo en ratas macho Sprague-Dawley con peso entre 200 y 250 $\mathrm{g}$ y edad de 8 a 10 semanas. Los animales se mantuvieron en el Bioterio de la Universidad del Valle en las siguientes condiciones: ciclo luzoscuridad de 12 horas; temperatura, $22^{\circ}$ a $26^{\circ} \mathrm{C}$, seis animales en cada jaula. El proyecto fue revisado y aprobado por el Comité de Ética Animal de la Universidad del Valle según consta en el acta No. 18 del 16 de julio de 2002.

\section{Evaluación de actividad diurética}

La evaluación de la diuresis se realizó de acuerdo a la técnica descrita por Lipschitz et al. (10). Se tomaron veinticuatro ratas y se repartieron en cuatro grupos de seis animales cada uno. Los animales se mantuvieron sin comida y sin agua en las 18 horas previas al inicio del experimento y durante las seis horas de recolección de la orina. Todos los tratamientos se administraron por vía oral con un volumen de $25 \mathrm{ml} / \mathrm{kg}$ del peso corporal del animal. El primer grupo recibió únicamente solución salina normal (sodio $=154$ $\mathrm{mmol} / \mathrm{L}$, cloro $=154 \mathrm{mmol} / \mathrm{L}$ ); el segundo grupo recibió furosemida $(10 \mathrm{mg} / \mathrm{kg}$ ) disuelta en solución salina normal; el tercero y el cuarto grupo recibieron $1 \mathrm{~g} / \mathrm{kg}$ y $2 \mathrm{~g} / \mathrm{kg}$ de $S$. scutellarioides, 
respectivamente. La orina se recolectó durante seis horas en jaulas metabólicas individuales a una temperatura ambiente de $22^{\circ} \mathrm{C}$, midiéndose la orina excretada a intervalos de una hora. Finalizado el experimento, se analizaron inmediatamente (en un tiempo menor de 30 minutos) las concentraciones de electrolitos $\left(\mathrm{Na}^{+}\right.$, $\mathrm{K}^{+}, \mathrm{Cl}^{-}$) en la orina recolectada; la determinación se realizó con la técnica de ion selectivo utilizando un analizador de electrolitos modelo Nova 10 de Biomedical ${ }$.

La excreción volumétrica de orina Urine volumetric excretion se calculó por medio de la siguiente fórmula: UVE=volumen recolectado/volumen administrado x 100.

\section{Análisis estadístico}

Los resultados de diuresis (UVE) se expresaron como la media del grupo y se procesaron con un análisis de varianza (ANOVA) de dos vías. Los datos de electrolitos en orina se expresaron como la media del grupo y se les realizó ANOVA de una vía. Las diferencias entre los grupos fueron analizadas con la prueba Bonferroni (UVE) y la de Neuman-Kleus (electrolitos en orina). Se aceptó $p<0,05$ como indicador de las diferencias estadísticamente significativas. Los datos fueron analizados, procesados y graficados por medio del programa GraphPad® Prism, versión 4.0, para Windows ${ }^{\circledR}$ XP. Los resultados obtenidos se exponen como el promedio del grupoterror estándar de la media.

\section{Resultados}

La administración de $S$. scutellarioides (1 y 2 g/ $\mathrm{kg}$ ) produjo un aumento significativo en la excreción volumétrica de orina (UVE) comparado con el grupo control (figura 1). El aumento en la UVE por 1 y $2 \mathrm{~g} / \mathrm{kg}$ de $S$. scutellarioides se manifestó principalmente a partir de la cuarta hora, con una UVE de $45,8 \pm 11,9$ y $46,3 \pm 4,5$, respectivamente, resultados éstos estadísticamente significativos $(p<0,01)$ en comparación con la UVE a la cuarta hora del grupo control $(15,2 \pm 5,2)$. En la segunda y tercera horas se observó una diferencia estadísticamente significativa $(p<0,05)$ en la diuresis obtenida con furosemida comparada con ambas dosis del extracto de $S$. scutellarioides.

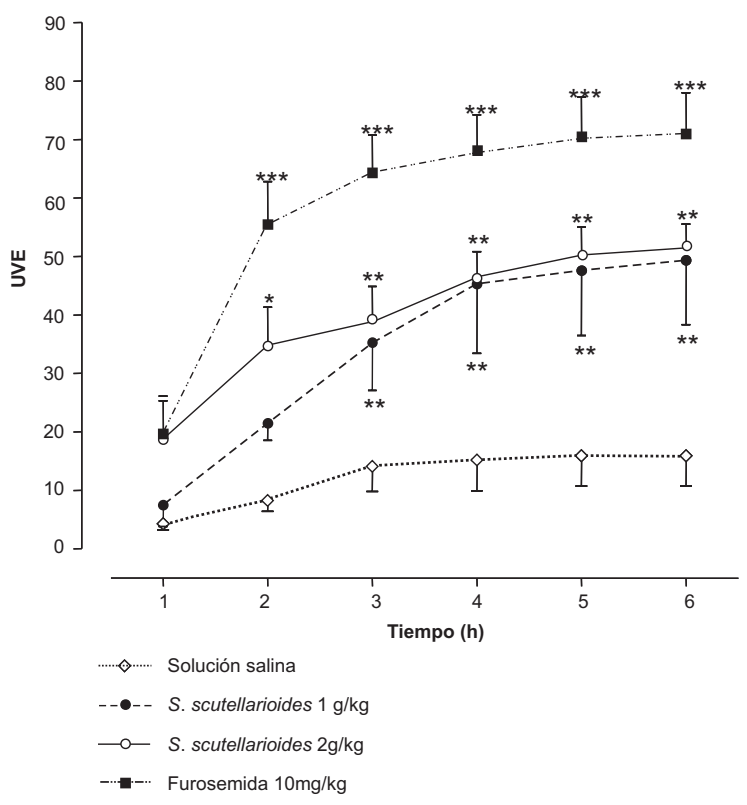

Figura 1. Efecto de $S$. scutellarioides en la excreción volumétrica de orina (UVE). La gráfica muestra la UVE de ratas machos tratados con solución salina, furosemida 10 $\mathrm{mg} / \mathrm{kg}$, S. scutellarioides $1 \mathrm{~g} / \mathrm{kg}$ y $2 \mathrm{~g} / \mathrm{kg}$. Los datos se presentan como el promedio \pm error estándar de la media. $\mathrm{n}=6 .{ }^{*} p<0,05 ;{ }^{* *} p<0,01 ;{ }^{* * *} p<0,001$ vs. grupo control.

Sin embargo, a partir de la cuarta hora no se encontraron diferencias estadísticamente significativas entre los grupos de S. scutellarioides y el grupo de furosemida (figura 1).

En la excreción de electrolitos en orina (cuadro 1), se encontró un aumento significativo en la excreción de sodio $(p<0,05)$, potasio $(p<0,001)$ y cloro $(p<0,001)$ en el grupo de $2 \mathrm{~g} / \mathrm{kg}$ de $S$. scutellarioides con respecto al grupo control. EI grupo de $1 \mathrm{~g} / \mathrm{kg}$ de $S$. scutellarioides mostró un aumento significativo en la excreción de potasio $(p<0,001)$ y cloro $(p<0,05)$. El grupo que recibió 2 $\mathrm{g} / \mathrm{kg}$ de $S$. scutellarioides presentó un aumento en la excreción de potasio y cloro $(p<0,05)$ en comparación con el grupo de $1 \mathrm{~g} / \mathrm{kg}$ de $S$. scutellarioides; no se encontraron diferencias estadísticamente significativas en la excreción de sodio entre los grupos.

Las ratas no presentaron cambios en su apariencia fisiológica durante el ensayo de diuresis, como tampoco en las 24 horas posteriores a la recolección de la orina. 
Cuadro 1. Efecto de la administración de $S$. scutellarioides en la excreción de electrolitos en orina

\begin{tabular}{lcccc}
\hline $\begin{array}{l}\text { Electrolitos } \\
(\mathbf{m E q} / \mathbf{L})\end{array}$ & Control & $\begin{array}{c}\text { Furosemida } \\
(\mathbf{1 0} \mathbf{~ m g / k g})\end{array}$ & \multicolumn{2}{c}{ S. scutellarioides $\mathbf{( g / k g})$} \\
\hline $\mathrm{Na}^{+}$ & $13,8 \pm 2,5$ & $14,3 \pm 1,7$ & $19,3 \pm 2,5$ & $24,7 \pm 3,8^{*}$ \\
$\mathrm{~K}^{+}$ & $26 \pm 1,76$ & $22,3 \pm 2,1$ & $55,5 \pm 8,3^{* * *}$ & $85,4 \pm 2^{* * *}$ \\
$\mathrm{Cl}^{-}$ & $48,7 \pm 2,3$ & $52,5 \pm 2,9$ & $66,2 \pm 5,6^{*}$ & $82,8 \pm 5,8^{* *}$ \\
\hline
\end{tabular}

Los datos se presentan como el promedio \pm error estándar de la media; $\mathrm{n}=6$.

${ }^{*} p<0,05 ;{ }^{* *} p<0,01 ;{ }^{* \star *} p<0,001$ vs. grupo control.

₹ UVE: volumen recolectado/volumen administrado $\times 100$.

\section{Discusión}

S. scutellarioides en dosis de 1 y $2 \mathrm{~g} / \mathrm{kg}$ mostró un efecto diurético significativo en comparación con el grupo control de solución salina normal (figura 1). La furosemida ocasionó un mayor efecto diurético comparada con $S$. scutellarioides, de significación estadística en la segunda y tercera horas de medición de la orina. A partir de la cuarta hora, las diferencias entre furosemida y $S$. scutellarioides no fueron significativas, posiblemente debido al limitado número de animales utilizados por grupo. La furosemida es un diurético de asa que ha sido utilizado ampliamente en este modelo de diuresis, incluidos varios estudios con especies vegetales $(11,12)$. El inicio de acción del efecto diurético de $S$. scutellarioides es retardado comparado con la furosemida, lo que puede obedecer a factores farmacocinéticos o farmacodinámicos de los principios activos de la planta.

Los efectos en la diuresis de $S$. scutellarioides en dosis de $1 \mathrm{~g} / \mathrm{kg}$ y $2 \mathrm{~g} / \mathrm{kg}$ fueron cuantitativamente similares. Sin embargo, la administración de la planta produjo un aumento dependiente de la dosis en la excreción de electrolitos, principalmente de potasio y cloro. Solamente se observó un incremento en la excreción de sodio con la dosis de $2 \mathrm{~g} / \mathrm{kg}$ de $S$. scutellariodes (cuadro 1). Los efectos en la excreción de electrolitos no pueden ser mediados por la concentración de potasio de la planta, debido a que se observó también una tendencia al aumento en la concentración de sodio urinario con la dosis más alta de $S$. scutellarioides (cuadro 1). Lo anterior se explica porque las concentraciones tubulares altas de potasio estimulan la actividad de la bomba $\mathrm{Na}^{+} / \mathrm{K}^{+}$ATPasa en la membrana basolateral de las células del epitelio tubular, disminuyendo la concentración de sodio en la orina (13). Además, el potasio altera el gradiente de voltaje transepitelial, favoreciendo la reabsorción de sodio $(14,15)$. Los datos de diuresis y excreción de electrolitos obtenidos en este estudio con la administración de $S$. scutellarioides son similares a los reportados con las tiacidas que actúan en el túbulo distal (16).

Recientemente se describió el efecto antihipertensivo de $S$. scutellarioides en ratas (Ramírez $\mathrm{JH}$, Palacios M, Gutiérrez O. Estudio del efecto antihipertensivo de la especie Salvia scutellarioides en un modelo de ratas hipertensas. Sometido para publicación en Colombia Médica, mayo de 2005). Sin embargo, la importancia del efecto diurético en la acción antihipertensiva de $S$. scutellarioides debe aclararse con futuros experimentos, ya que el efecto farmacológico de una planta se puede explicar por la acción aditiva o la sinergia de varios principios activos (17).

Se requieren más estudios con el objetivo de determinar los mecanismos de acción y toxicidad, y aislar los principios activos de $S$. scutellarioides. Además, se deben realizar estudios sobre la interacción de la planta con medicamentos de uso frecuente en la práctica médica colombiana, dado que un segmento importante de la población consume fitofármacos con la creencia común que no presentan efectos adversos ni interacciones medicamentosas. La continuación de estos estudios se justifica por el amplio uso de la planta entre la población colombiana y por el desconocimiento de su eficacia sobre los tratamientos convencionales para la hipertensión arterial.

En conclusión, el extracto acuoso de $S$. scutellarioides en dosis de 1 y $2 \mathrm{~g} / \mathrm{kg}$ produjo un 
aumento significativo en la diuresis de potasio y cloro con respecto al grupo control de solución salina. Este estudio corrobora la actividad diurética de S. scutellarioides reportada por los practicantes de la medicina tradicional colombiana.

\section{Conflicto de intereses}

Los autores manifiestan que sus ingresos provienen de la práctica docente en la Universidad del Valle.

\section{Financiación}

La financiación de esta investigación se obtuvo mediante el proyecto de recuperación contingente con código 11060413079 existente entre la Universidad del Valle y el Instituto Colombiano para el Desarrollo de la Ciencia y la Tecnología, Colciencias.

\section{Referencias}

1. García H. Familia Lamiaceae. Flora Medicinal de Colombia. Bogota: Tercer Mundo Editores; 1992. p.33-4.

2. Piñeros CJ, García HB, Iregui AB, Prias EL, Perdomo C. Farmacocinética y farmacodinamia de los preparados naturales. Plantas medicinales: compendio de farmacología vegetal. Bogota: Fedicor; 1991. p.42-83.

3. Colmenares AJ, Ramírez AB. Salvia scutellarioides. Treinta plantas medicinales del Valle del Cauca, fundamentos fitoquímicos y farmacológicos que sustentan sus usos. Cali: Feriva; 2001. p.49-50.

4. Bernal HY, Correa JE. Salvia palaefolia. Especies vegetales promisorias en los países del convenio Andrés Bello. Bogotá: SECAB; 1994. p.1-156.

5. Ahmed AA, Castro B. Sesquiterpene lactones from Salvia palaefolia. Phytochemistry 1990;11:3581-5.

6. Matsubara T, Bohgaki T, Watarai M, Suzuki H, Ohashi K, Shibuya H. Antihypertensive actions of methylripariochromene A from Orthosiphon aristatus, an Indonesian traditional medicinal plant. Biol Pharm Bull 1999;22:1083-8.
7. Ohashi K, Bohgaki T, Shibuya H. Antihypertensive substance in the leaves of kumis kucing (Orthosiphon aristatus) in Java Island. Yakugaku Zasshi 2000;120:474-82.

8. Ohashi K, Bohgaki T, Matsubara T, Shibuya H. Indonesian medicinal plants. XXIII. Chemical structures of two new migrated pimarane-type diterpenes, neoorthosiphols $A$ and $B$, and suppressive effects on rat thoracic aorta of chemical constituents isolated from the leaves of Orthosiphon aristatus (Lamiaceae). Chem Pharm Bull 2000;48:433-5.

9. Sharapin N. Materias primas vegetales para la industria de productos fitoterapéuticos. En: Pinzón RS, editor. Fundamentos de tecnología de productos fitoterapéuticos. Bogotá:: CYTED Convenio Andrés Bello; 2000. p.17-26.

10. Lipschitz WL, Haddian Z, Kerpscar A. Bioassay of diuretics. J Pharm Exp Ther 1943;79:110-6.

11. Camargo ME, Berdeja B, Miranda G. Diuretic effect of the aqueous extract of Bidens odorata in the rat. $J$ Ethnopharmacol 2004;95:363-6.

12. Maghrani M, Zeggwagh NA, Haloui M, Eddouks M. Acute diuretic effect of aqueous extract of Retama raetam in normal rats. J Ethnopharmacol 2005;99:315.

13. Feraille E, Doucet A. Sodium-potassium-adenosinetriphosphatase-dependent sodium transport in the kidney: hormonal control. Physiol Rev 2001;81:345418.

14. Burg MB. Tubular chloride transport and the mode of action of some diuretics. Kidney Int 1976;9:189-97.

15. Schlueter W, Keilani T, Hizon M, Kaplan B, Batlle DC. On the mechanism of impaired distal acidification in hyperkalemic renal tubular acidosis: evaluation with amiloride and bumetanide. J Am Soc Nephrol 1992;3:953-64.

16. Masereeuw R, Moons WM, Russel FG. Saturable accumulation and diuretic activity of hydrochlorothiazide in the isolated perfused rat kidney. Pharmacology 1997;54:33-42.

17. Spinella M. The importance of pharmacological synergy in psychoactive herbal medicines. Altern Med Rev 2002;7:130-7. 